\title{
67P/Churyumov-Gerasimenko at large heliocentric distance ${ }^{\star}, \star \star$
}

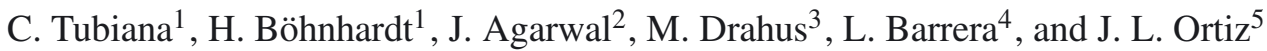 \\ ${ }^{1}$ Max-Planck-Institut für Sonnensystemforschung, Max-Planck-Str. 2, 37191 Katlenburg-Lindau, Germany \\ e-mail: tubiana@mps.mpg.de \\ 2 European Space Research and Technology Centre, 2200 AG Noordwijk, The Netherlands \\ 3 Department of Earth and Space Sciences, University of California at Los Angeles, Los Angeles, CA 90095-1567, USA \\ ${ }^{4}$ Universidad Metropolitana de Ciencias de la Educación, Santiago, Chile \\ ${ }^{5}$ Instituto de Astrofísica de Andalucía, CSIC, Granada, Spain
}

Received 29 October 2010 / Accepted 9 December 2010

\section{ABSTRACT}

\begin{abstract}
Aims. The Jupiter family comet (JFC) 67P/Churyumov-Gerasimenko (67P/C-G) is the target of ESA's ROSETTA mission. Observational campaigns and theoretical investigations were performed to characterise 67P/C-G in terms of nucleus properties (e.g. size, surface colours, rotational period), gas and dust production, and plasma environment in preparation for the rendezvous with the spacecraft; however, there are still open questions that need to be addressed. Our observations of 67P/C-G are important not only for a good planning of the rendezvous of the ROSETTA spacecraft with $67 \mathrm{P} / \mathrm{C}-\mathrm{G}$, but also for providing valuable information on the basic physical properties of the nuclei of JFCs at large heliocentric distances. Moreover, this information will help to identify to what extent $67 \mathrm{P} / \mathrm{C}-\mathrm{G}$ is a typical JFC.

Methods. We performed broad-band imaging and low-resolution spectroscopy of 67P/C-G in the visible wavelength range during five periods between April 2004 and July 2007 at the ESO Very Large Telescope (VLT) with the FORS2 instrument. At the time of the observations 67P/C-G was at heliocentric distance $r \geq 4.6 \mathrm{AU}$. The imaging data were used to search for a faint coma, to improve the phase function of the nucleus, to constrain its rotational period, and to analyse the neck-line of dust close to the nucleus.

Results. The comet appears point-like and no coma signature was found around the nucleus. The most realistic representative of the phase function of $67 \mathrm{P} / \mathrm{C}-\mathrm{G}$ is the linear approximation: This could be interpreted to mean that the opposition effect is not very pronounced for $67 / \mathrm{C}-\mathrm{G}$ in the phase angle range between $0.5^{\circ}$ and $10^{\circ}$. We determined that the magnitude dependence on the phase angle is very steep, with the linear phase coefficient in the range $\beta=0.061-0.076 \mathrm{mag} /{ }^{\circ}$. The colour indices and reflectance spectrum show that the nucleus of $67 \mathrm{P} / \mathrm{C}-\mathrm{G}$ is slightly redder than the Sun (spectral slope $\sim 11 \% / 1000 \AA$ ) and uniform with the rotational phase. A tail-like structure of heavy grains is detected in April 2004, June 2004, and May 2006. Based on its orientation and the variations of its surface brightness we interpreted it as a neck-line phenomenon.
\end{abstract}

Key words. comets: general - comets: individual: 67P/Churyumov-Gerasimenko - methods: observational - techniques: photometric - techniques: spectroscopic

\section{Introduction}

The Jupiter family comet 67P/Churyumov-Gerasimenko $(67 \mathrm{P} / \mathrm{C}-\mathrm{G})$ is the target of ESA's ROSETTA mission (Schwehm \& Schulz 1998). What makes this mission exceptional is that the spacecraft will follow the comet in its journey towards the Sun, examining how a frozen comet is transformed by solar heating and space weather. In 2003, owing to a delay in the launch of the ROSETTA spacecraft, 67P/C-G was chosen on the basis of orbital considerations as the most suitable alternative to the original target, $46 \mathrm{P} /$ Wirtanen, even though very little was known at that time about this comet and the physical properties of its nucleus. Since then, observational campaigns and theoretical investigations were performed to establish a detailed portrait of $67 \mathrm{P} / \mathrm{C}-\mathrm{G}$ in preparation for the rendezvous with the spacecraft, which will take place in 2014. Because of repeated close encounters with Jupiter, the orbital evolution

* Based on observations performed at the European Southern Observatory, Chile (ESO Programmes 073.C-0346, 077.C-0609, 277.C-5038, 079.C-0687(C) \& 080.C-0259(B)).

$\star \star$ Table 5 is only available in electronic form at the CDS via anonymous ftp to cdsarc.u-strasbg.fr $(130.79 .128 .5)$ or via http://cdsarc.u-strasbg.fr/viz-bin/qcat?J/A+A/527/A113 of $67 \mathrm{P} / \mathrm{C}-\mathrm{G}$ is chaotic. The last encounter in February 1959 produced a drastic change in the comet's orbit and may have triggered its activity, which most likely led to its discovery in 1969 (Lamy et al. 2007).

Thanks to space- and ground-based observations performed in different wavelength regions during the last orbit, an initial characterization of $67 \mathrm{P} / \mathrm{C}-\mathrm{G}$ has been drawn (see Lamy et al. 2007; Agarwal et al. 2007; Hansen et al. 2007; Lowry et al. 2006; Tubiana et al. 2008; Agarwal et al. 2010; Kelley et al. 2009). However, there are still open questions that need to be addressed.

Here we present new results of visible imaging and spectroscopy of 67P/C-G observed at the ESO Very Large Telescope (VLT), obtained by combining the dataset presented in Tubiana et al. (2008) (June 2004, May 2006, and August 2006) with additional datasets acquired in April 2004 and July 2007. This extended dataset is used to monitor the dust environment of $67 \mathrm{P} / \mathrm{C}-\mathrm{G}$ in the aphelion arc from 4.7 AU pre-aphelion to 4.6 AU post-aphelion and to characterise the comet's nucleus. Since no activity was detected, we focused our analysis mainly on the characterisation of the nucleus of $67 \mathrm{P} / \mathrm{C}-\mathrm{G}$, in particular on the determination of the phase function of the nucleus and its rotational period, and its tail-like structure. Our observations are 
Table 1. Details of the observations of 67P/C-G.

\begin{tabular}{|c|c|c|c|c|c|c|c|}
\hline Start time [UT] & End time [UT] & $r[\mathrm{AU}]$ & $\Delta[\mathrm{AU}]$ & $\alpha\left[^{\circ}\right]$ & $\mathrm{GL}\left[{ }^{\circ}\right]$ & Sky condition & Observation type \\
\hline $2004-04-1500: 58$ & $15.04 .0408: 40$ & 4.67 & 3.68 & 2.1 & 47.4 & CLR/non PHO & IM \\
\hline 2004-06-16 23:201 & $17.06 .0402: 17$ & 4.89 & 4.29 & 10.3 & 52.6 & CLR & IM \\
\hline $2006-05-2601: 00^{1}$ & $26.05 .0609: 42$ & 5.62 & 4.61 & 1.3 & 14.7 & CLR & IM \& SP \\
\hline $2006-05-3100: 28^{1}$ & $31.05 .0609: 33$ & 5.61 & 4.60 & 0.6 & 15.3 & CLR & IM \& SP \\
\hline 2006-06-01 00:17 & 01.06.06 09:46 & 5.61 & 4.60 & 0.5 & 15.5 & CLR & IM \& SP \\
\hline 2006-08-18 01:421 & $18.08 .0602: 03$ & 5.51 & 5.27 & 10.5 & 21.3 & CLR & IM \\
\hline 2006-08-19 01:291 & $19.08 .0601: 48$ & 5.51 & 5.28 & 10.5 & 21.2 & CLR & IM \\
\hline $2006-08-2302: 32^{1}$ & $23.08 .0602: 52$ & 5.50 & 5.34 & 10.6 & 21.1 & CLR & IM \\
\hline $2006-08-2323: 30^{1}$ & $23.08 .0623: 53$ & 5.50 & 5.35 & 10.6 & 21.1 & CLR & IM \\
\hline $2007-07-1623: 51$ & 17.07 .0708 .00 & 4.63 & 3.72 & 6.0 & 0.6 & CLR & IM \& SP \\
\hline
\end{tabular}

Notes. $r$ and $\Delta$ are the heliocentric and geocentric distances, respectively, $\alpha$ is the phase angle, and GL the galactic latitude of the comet. The average nightly sky conditions are reported $(\mathrm{PHO}=$ photometric, $\mathrm{CLR}=$ clear) as well as the type of observations performed $(\mathrm{IM}=\mathrm{imaging}$, $\mathrm{SP}=$ spectroscopy$).^{(1)}$ From Tubiana et al. (2008).

not only important for a good planning of the rendezvous of the ROSETTA spacecraft with the comet, but will also provide valuable information on the basic physical properties of the nucleus of JFCs. Moreover, this information will help to identify to what extent $67 \mathrm{P} / \mathrm{C}-\mathrm{G}$ is a typical Jupiter family comet (JFC), comparing our results (e.g. rotational period, phase function, nucleus colors) with the average values determined by Lamy et al. (2009) and Snodgrass et al. (2011) for JFCs.

\section{Observations and data reduction}

We observed 67P/C-G in the visible wavelength range between April 2004 and July 2007 with the FORS2 instrument at the 8.2 m Very Large Telescope on Cerro Paranal (Chile). The observational circumstances are summarised in Table 1. FORS2 (Appenzeller et al. 1998) is the visual and near UV FOcal Reducer and low-dispersion Spectrograph for the VLT of the European Southern Observatory (ESO). Its detector consists of a mosaic of two $2 \mathrm{k} \times 4 \mathrm{k}$ MIT CCDs ( $15 \mu \mathrm{m}$ pixels). In the standard resolution mode, which we used for the observations, the image scale in the default readout mode $(2 \times 2$ pixel binning $)$ is $0.25^{\prime \prime} /$ pix, providing a field of view of $6.8^{\prime} \times 6.8^{\prime}$. The observations were performed with the telescope tracking at the proper motion rate of the comet.

In the subsequent paragraphs the April 2004 and July 2007 datasets are described, while the other three datasets are described in Tubiana et al. (2008).

\subsection{Observations in April 2004}

We observed 67P/C-G for one full night on April 15, 2004. The main purpose of this observing run was to determine the $67 \mathrm{P} / \mathrm{C}$ $\mathrm{G}$ nucleus light curve, its size, axis-ratio and colours. The dataset is composed of a total of 50 broadband images of $67 \mathrm{P} / \mathrm{C}-\mathrm{G}$ : 7 in $V$ Bessell filter $\left(\lambda_{\text {central }}, V=5450 \AA\right), 30$ in the $R$ special filter $\left(\lambda_{\text {central }, R}=6380 \AA\right)$, and 13 in $I$ Bessell filter $\left(\lambda_{\text {central }, I}=\right.$ $7970 \AA$ ) $)$. To ensure a good signal-to-noise ratio $(S / N \sim 50)$, we used an exposure time of $300 \mathrm{~s}$ for the $V$ and $R$ filter images; in order to avoid saturation of the CCD by sky background light, the $I$ filter images were split into two exposures of $150 \mathrm{~s}$ each.

The images were reduced using standard techniques of bias subtraction, flat field correction (using a super-flat field created from the night exposures of the comet), exposure time normalisation, and sky background subtraction. Because the night of observation was not photometric, we observed the same star field in July 2007 and used this to cross-calibrate.

\subsection{Observations in July 2007}

One full night of observation was granted on July 17, 2007 during which we performed broadband photometry and long slit spectroscopy of $67 \mathrm{P} / \mathrm{C}-\mathrm{G}$ to study the dust environment of the comet at large heliocentric distance, improve the nucleus phase function and its rotational period, and to look for possible colour variations with the rotational phase.

Photometry: A total of 156 images of $67 \mathrm{P} / \mathrm{C}-\mathrm{G}$ were taken: three in $B$ Bessell filter $\left(\lambda_{\text {central }}, B=4290 \AA\right)$, three in $V$ Bessell filter $\left(\lambda_{\text {central }, V}=5450 \AA\right), 143$ in the $R$ special filter $\left(\lambda_{\text {central }, R}=\right.$ $6380 \AA)$, and seven in $I$ Bessell filter $\left(\lambda_{\text {central }, I}=7970 \AA\right)$. To ensure a reasonably good signal-to-noise ratio $(S / N>50)$ we used an exposure time of $100 \mathrm{~s}$ in $R$ and $V$ filters, $140 \mathrm{~s}$ in $I$ filter and $420 \mathrm{~s}$ in $B$ filter.

Bias, flat fields, and standard star fields were taken each night. Standard techniques of image reduction were applied, as described in Sect. 2.1. For the flux calibration we determined the zero points and extinction coefficients from the various standard star fields observed at different air mass during the same night. Regarding the colour coefficients, we adopted those determined in May 2006 by Tubiana et al. (2008) for $V, R, I$ filters, while for the $B$ filter we used the value provided by ESO for the night when the observation was carried out (http://archive.eso.org/bin/qc1_cgi? action=qc1_browse_instrume-instrume=FORS2).

The comet was at low galactic latitude $\left(\sim 0.6^{\circ}\right)$, thus in front of a star-rich background. Owing to its motion, $67 \mathrm{P} / \mathrm{C}-\mathrm{G}$ passed in the vicinity of plenty of stars. To remove the stars' contamination we rebinned each image to a new pixel size of $0.2 \times 0.2$ pixel, then we aligned the images on a star, and finally we subtracted two rebinned aligned images to obtain a final frame in which the comet was free from contaminations.

Spectroscopy: Reflectance spectra of the comet were obtained using a 1.3" slit and the low-resolution grism 150I+27 $(\Delta \lambda=3500-11000 \AA$, dispersion $=3.45 \AA /$ pix $)$. The slit of the spectrograph was placed parallel to the direction of proper motion of the comet. For calibration purposes, spectra of solar analogue stars were taken at similar airmasses and with the instrument in the same configuration as for the comet's spectra. Standard methods for spectroscopy reduction and extraction 


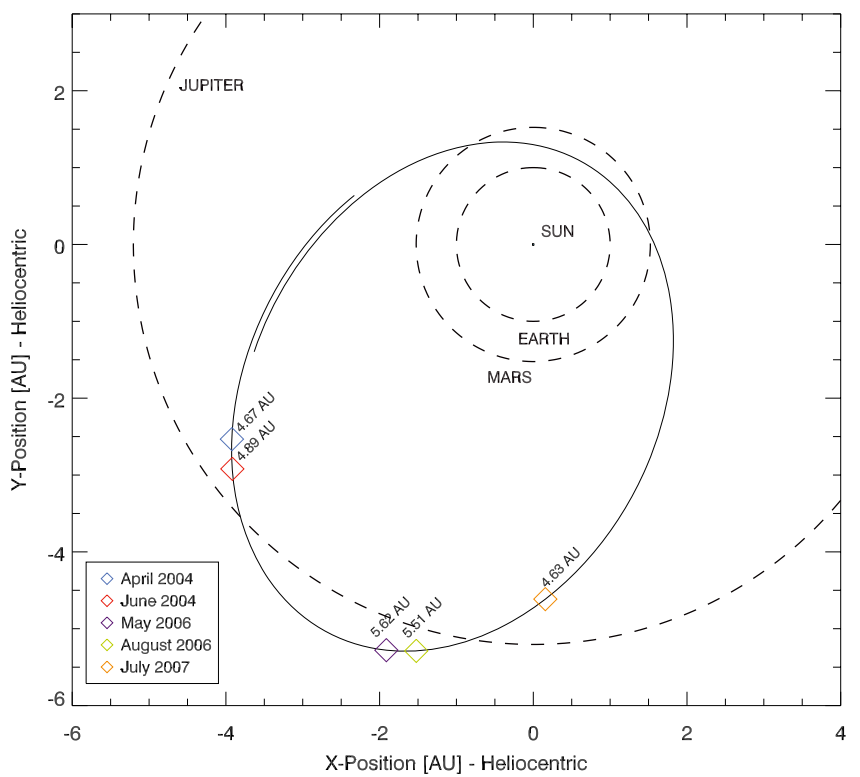

Fig. 1. Orbital positions of $67 \mathrm{P} / \mathrm{C}-\mathrm{G}$ at the time of the observations. The orbits of Mars, the Earth, and Jupiter are displayed.

were applied (bias subtraction, flat fielding using lamp flats, wavelength calibration through arclamp spectra, exposure time normalisation and sky background subtraction). To remove the solar spectrum from that of the comet, we divided the comet spectra by the average of the solar analogue spectra obtained with the same slit. Finally, the comet spectrum was normalised to unity at $6380 \AA$ (central wavelength of the $R$ filter).

\subsection{Photometry}

Using normal aperture photometry, we measured the apparent magnitude of 67P/C-G in each frame, from which we calculated the reduced magnitude $\mathrm{m}(1,1, \alpha)$ normalised to the Sun and Earth distances equal to $1 \mathrm{AU}$. The apparent magnitude from April 2004 and July 2007 is listed in Table 5, while the photometry from June 2004, May 2006, and August 2006 is listed in Table 5 of Tubiana et al. (2008). The observing time is corrected for the light travel time. For the estimate of the photometric error, we considered the error on the aperture measurement, the error of the flux calibration coefficients, and errors from the basic data reduction process (mostly the "non-flatness" of the flat field used). The final error on the magnitudes was obtained by the Gaussian error propagation.

\section{Results}

Figure 1 shows the locations of $67 \mathrm{P} / \mathrm{C}-\mathrm{G}$ at the time of observations.

Examples of comet images in $R$ filter for each of our observing runs are displayed in Fig. 2. Each panel displays only part of the entire field of view. In May 2006, August 2006, and July 2007 67P/C-G was at low galactic latitude, thus in front of a star-rich background.

We investigated the presence of coma around the nucleus with the methods described in Tubiana et al. (2008): For each observing epoch $67 \mathrm{P} / \mathrm{C}-\mathrm{G}$ appears like a point source, indicating that no coma is evident around the nucleus at heliocentric distances $r \geq 4.6$ AU. Using an individual image of the May 2006 dataset, we determined a 3- $\sigma$ upper limit for the dust production rate of $\operatorname{Af} \rho \sim 1.94 \mathrm{~cm}$, which corresponds to a dust production of $Q_{\mathrm{d}} \sim 0.22 \mathrm{~kg} / \mathrm{s}$ using a simple heuristic relationship by Kidger (2004).

Because no activity was detected around the nucleus of $67 \mathrm{P} / \mathrm{C}-\mathrm{G}$, we focused our analysis mainly on the characterisation of the properties of the nucleus of $67 \mathrm{P} / \mathrm{C}-\mathrm{G}$ and of its taillike structure.

\subsection{Nucleus properties}

\subsubsection{Phase function and rotational period}

Brightness changes in the reduced magnitude $\mathrm{m}(1,1, \alpha)$ over the long period of our observations of $67 \mathrm{P} / \mathrm{C}-\mathrm{G}$ are induced by the nucleus rotation and the phase darkening. Thus, to have valuable information on both effects, the phase darkening and the nucleus rotation have to be considered and determined simultaneously.

For this purpose, we used the observations of $67 \mathrm{P} / \mathrm{C}-\mathrm{G}$ performed in May 2006, August 2006, and July 2007. While the latter dataset is described in Sect. 2.2, the other two datasets are described in Tubiana et al. (2008), who used them for a first simultaneous determination of the rotational period and phase coefficient of 67P/C-G. In July 2007 the comet was at phase angle of about $6^{\circ}$, intermediate between the phase angle in May 2006 and August 2006 (see Table 1), which makes it especially important in the determination of the phase function of the comet. Moreover, the combined dataset covers a much longer time interval compared to the one used by Tubiana et al. (2008), which allows for higher accuracy in the rotational period and phase coefficient. While combining datasets from different epochs brings these advantages, caution has to be taken. Indeed because of the orbital motion of the comet, changes of the viewing geometry can occur, which can produce non-negligible changes of the mean magnitude, amplitude, and shape of the light curve. Following the approach of Drahus \& Waniak (2006), who adopted the routine by Michalowski (1988), we calculated that the maximum phase shift induced by changes of the observing geometry of 67P/C-G between May 2006 and July 2007 is 0.13 at $95 \%$ confidence level and between the May 2006 and August 2006 observations is less than 0.01 at $95 \%$ confidence level. All these phase shifts seem negligibly small, and therefore also the other light curve parameters should not have changed considerably. In contrast, the maximum phase shift between May 2006 and April 2004 runs is 0.26 at $95 \%$ confidence level, which is the reason why we did not include it in the analysis.

As input data we used the reduced magnitude $\mathrm{m}(1,1, \alpha)$. The May 2006 dataset consists of 95 data points, the August 2006 of 12, and the July 2007 one of 126. Thus, the combined dataset May 2006 + August 2006 + July 2007 consists of 233 data points.

The method we used for the simultaneous determination of rotational period and phase coefficient is described in Tubiana et al. (2008). We restricted the ranges of free parameters (frequency of periodicity, linear phase coefficient and slope parameter) to those where the solutions were found by Tubiana et al. (2008). Thus, we scrutinised the frequency $f$ in the range $\Delta f=0.077-0.080 \mathrm{~h}^{-1}$, the linear phase coefficient $\beta$ in the range $\Delta \beta=0.04-0.10 \mathrm{mag} /{ }^{\circ}$ and the slope parameter $G$ in the range $\Delta G=-0.15-+0.45$. The phase dispersion minimisation (PDM) algorithm was used with the number of bins $N_{\mathrm{b}}=25$ and the number of covers $N_{\mathrm{c}}=5$, whereas the $\chi^{2}$ minimisation method was run for the number of frequencies (i.e. number of harmonics including the base frequency) $N_{\mathrm{f}}=5$. Both the PDM and the $\chi^{2}$ minimisation methods returned a number of possible 


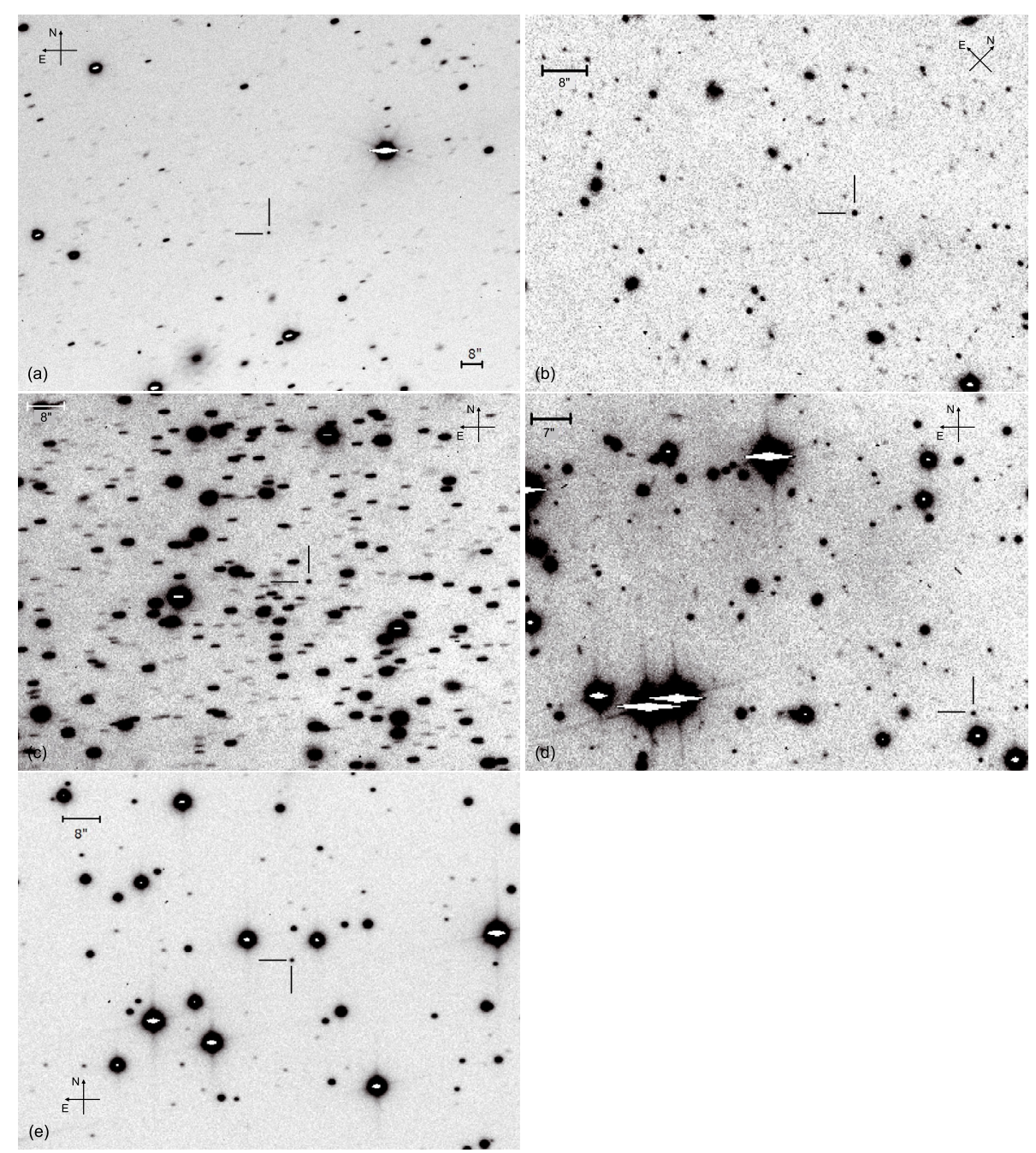

Fig. 2. $R$ filter images of 67P/C-G taken in April 2004 a), June 2004 b), May 2006 c), August 2006 d), and July 2007 e). Image scale and orientation are shown. The position of the comet in the frames is marked. Uneven diffuse background is caused by galactic clouds.

solutions for the rotational period and phase coefficient of 67P/C-G. In Fig. 3 we present the adaptive cross-cuts through the periodograms, where $\Theta$ is displayed against frequency for the optimum phase function.

The quality of the periodogram, indicated by the $\Theta$ parameter, obtained with the linear approximation of the phase function is systematically better than the one obtained from the IAUadopted phase law. We conclude that the linear approximation best represents the phase function of $67 \mathrm{P} / \mathrm{C}-\mathrm{G}$. Thus, from now on, we focus our attention only on the results obtained assuming the linear phase function approximation.

Figure 3 shows a number of possible solutions for rotational period and linear phase coefficient. Each solution has high precision thanks to the long time interval covered by the combined dataset. However, the uneven sampling of the time interval does not allow us to distinguish among the possible solutions. Table 2 summarises the best solutions for rotational period and linear phase coefficient of 67P/C-G. Because the PDM and the $\chi^{2}$ minimisation methods returned per each solution very close values of rotational period and linear phase coefficient, we determined for each solution their mean values. Based on the values of the linear phase coefficient, the solutions displayed in Table 2 can be divided into two groups. Group 1 (solutions 1, 2, 4, 5, 8, 9) represents a steeper dependence of brightness with phase angle, while in group 2 (solutions $3,6,7$ ) the dependence is shallower.
The linear phase coefficients in group 1 coincide within the uncertainties with the $\beta$ value determined by Tubiana et al. (2008). Examples of rotational phase profiles are displayed in Fig. 4.

The minima in the periodogram (Fig. 3) are equally wide, which indicates that the uncertainties in the rotational period and linear phase coefficient are comparable for all solutions. Thus, we calculated the errors only for one solution (solution 9) and adopted them for all the other solutions. Moreover, since the PDM and the $\chi^{2}$ minimisation methods returned per each solution very close values of rotational period and linear phase coefficient, for the determination of the uncertainties we consider their mean values.

The intrinsic precision of the period estimation is improved when considering also the July 2007 dataset, but, unfortunately, a discrimination among solutions is not uniquely possible. This is because of the large time gap between the different datasets used for the analysis. Thus, we can only conclude that the rotational period and linear phase coefficient of $67 \mathrm{P} / \mathrm{C}-\mathrm{G}$ are in the range $T_{\text {rot }}=12.6801-12.8612 \mathrm{~h}$ and $\beta=0.061-0.076 \mathrm{mag} /{ }^{\circ}$, respectively.

The overall shape of the rotational phased light curve is similar for all used rotational periods and linear phase coefficients. $67 \mathrm{P} / \mathrm{C}-\mathrm{G}$ shows an asymmetric double peak profile. The brighter peak is wider than the secondary one and presents a modulation 

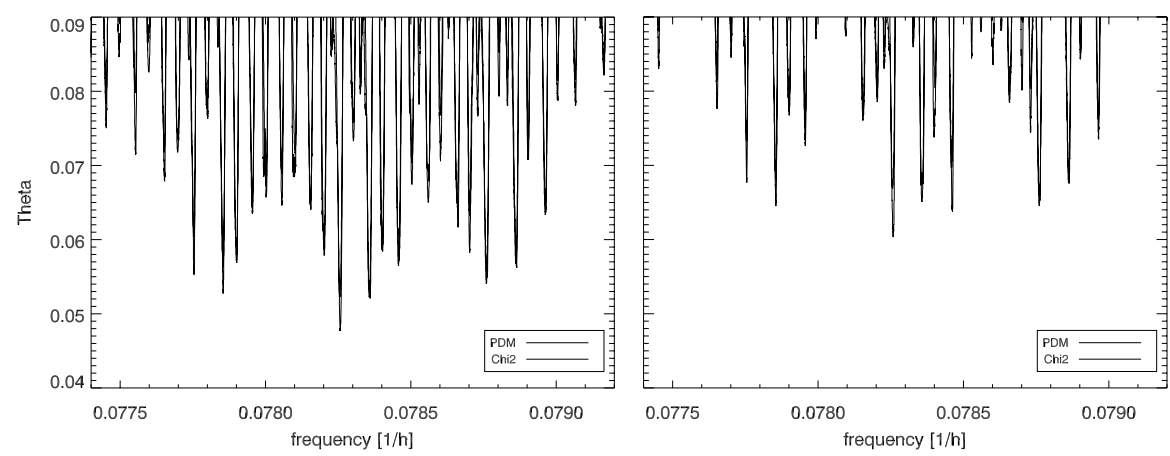

Fig. 3. Adaptive cross-cuts through the periodograms for the PDM and the $\chi^{2}$ methods using the linear approximation for the phase function (left panel) and the IAU-adopted (Bowell et al. 1989) one (right panel).

Table 2. Best solutions for the rotational period and linear phase function coefficient $\beta$ of 67P/C-G using the May 2006, August 2006, and July 2007 photometry of the nucleus.

\begin{tabular}{lcccccc}
\hline \hline Solution ID & $T_{\text {rot }, P D M}[\mathrm{~h}]$ & $T_{\text {rot, } \chi^{2}}[\mathrm{~h}]$ & $\beta_{P D M}\left[\mathrm{mag} /{ }^{\circ}\right]$ & $\beta_{\chi^{2}}\left[\mathrm{mag} /{ }^{\circ}\right]$ & $T_{\text {rot,mean }}[\mathrm{h}]$ & $\beta_{\text {mean }}\left[\mathrm{mag} /{ }^{\circ}\right]$ \\
\hline Solution 1 & 12.6805 & 12.6801 & 0.075 & 0.073 & $12.6803 \pm 0.0002$ & $0.074 \pm 0.002$ \\
Solution 2 & 12.6968 & 12.6964 & 0.074 & 0.072 & $12.6966 \pm 0.0002$ & $0.073 \pm 0.002$ \\
Solution 3 & 12.7546 & 12.7546 & 0.063 & 0.063 & $12.7546 \pm 0.0002$ & $0.063 \pm 0.002$ \\
Solution 4 & 12.7621 & 12.7617 & 0.075 & 0.075 & $12.7619 \pm 0.0002$ & $0.075 \pm 0.002$ \\
Solution 5 & 12.7786 & 12.7785 & 0.075 & 0.073 & $12.7786 \pm 0.0002$ & $0.074 \pm 0.002$ \\
Solution 6 & 12.7874 & 12.7875 & 0.064 & 0.064 & $12.7875 \pm 0.0002$ & $0.064 \pm 0.002$ \\
Solution 7 & 12.8370 & 12.8368 & 0.062 & 0.061 & $12.8369 \pm 0.0002$ & $0.062 \pm 0.002$ \\
Solution 8 & 12.8446 & 12.8446 & 0.073 & 0.073 & $12.8446 \pm 0.0002$ & $0.073 \pm 0.002$ \\
Solution 9 & 12.8612 & 12.8611 & 0.074 & 0.075 & $12.8612 \pm 0.0002$ & $0.075 \pm 0.002$ \\
\hline
\end{tabular}

Notes. $T_{\text {rot,mean }}$ and $\beta_{\text {mean }}$ are the mean values of the rotational periods $\left(T_{\text {rot }}\right)$ and linear phase coefficients $(\beta)$, respectively, determined with the PDM method and the $\chi^{2}$ minimisation method.

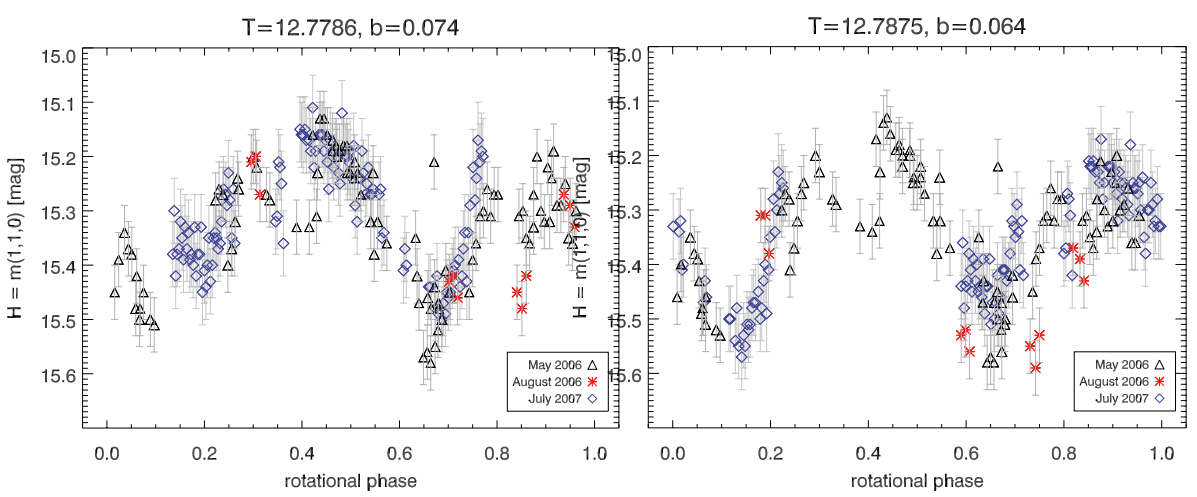

Fig. 4. Rotational phase profiles for solutions 5 and $6\left(T_{\text {rot,mean }}, \beta_{\text {mean }}\right)$, summarised in Table 2 , representative of solutions belonging to group 1 and group 2, respectively. The overall shape of the light curve is similar for all determined rotational periods and linear phase coefficients. that is not visible in the secondary peak. The minima in the light curve are narrow.

We determined the absolute magnitude $\mathrm{m}(1,1,0)$ of the April 2004 and June 2004 runs using $\beta=0.076 \mathrm{mag} /{ }^{\circ}$ and $\beta=$ $0.063 \mathrm{mag} /{ }^{\circ}$. The partial light curves from the April 2004 and June 2004 observations are overplotted on the previously determined phase profiles of $67 \mathrm{P} / \mathrm{C}-\mathrm{G}$, after they were arbitrarily shifted in the rotational phase to best match (estimated by eye) the rotational light curve (Fig. 5).

The difference between the two phase profiles results mainly from the June 2004 and August 2006 datasets. Because they were observed at a phase angle of about $10^{\circ}$, they are most sensitive to changes of the linear phase coefficient.

If we assume that the light curve did not change over the orbital motion of the comet, the three solutions belonging to group 2 display the August 2006 and June 2004 datasets systematically offset with respect to the May 2006 and July 2007 datasets. Thus, under this hypothesis, the aforementioned solutions look less realistic than the others, and they might be rejected: The brightness dependence on phase angle is very steep and is not well reproduced by the linear phase coefficient of $\beta=0.063 \mathrm{mag} /{ }^{\circ}$. Ignoring the hypothesis of no light curve changes over the orbital motion, a discrimination among solutions is not uniquely possible.

We consider the linear approximation the more realistic representation of the phase function of $67 \mathrm{P} / \mathrm{C}-\mathrm{G}$ : It could be interpreted in a way that for $67 / \mathrm{C}-\mathrm{G}$, the opposition effect is not very pronounced, if present at all, in the phase angle range between $0.5^{\circ}$ and $10^{\circ}$. This conclusion is also supported by the behaviour of the reduced magnitude $m(1,1, \alpha)$ versus phase angle, as shown in Fig. 6, where the mid-point $R$ filter reduced magnitude is plotted versus phase angle. The mid-point magnitude is defined as the mean value between the maximum and the minimum magnitude of the light curve. The measurements obtained in April 2004 and July 2007, when 67P/C-G was at a phase angle of about $\sim 2^{\circ}$ and $\sim 6^{\circ}$, respectively, show that the brightness dependence on phase angle is linear in the range between $0.5^{\circ}$ and $10^{\circ}$, suggesting that $67 \mathrm{P} / \mathrm{C}-\mathrm{G}$ does not show a significant opposition effect in this phase angle range. The slopes corresponding to a linear phase coefficient $\beta=0.076 \mathrm{mag} /{ }^{\circ}$, representative for 

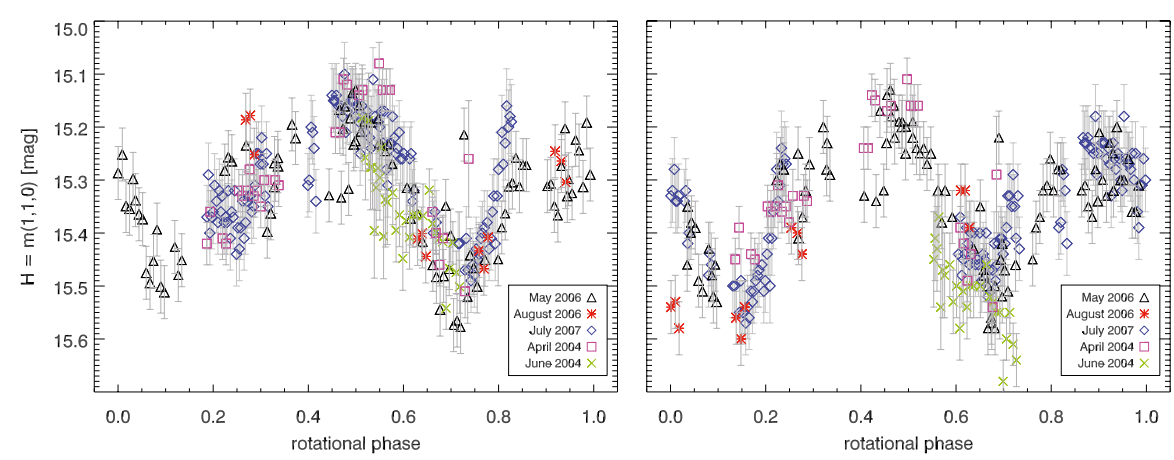

Fig. 5. Phased light curves of $67 \mathrm{P} / \mathrm{C}-\mathrm{G}$ representative of group 1 (left panel) and group 2 (right panel), overplotted with the observations of April 2004 and June 2004 phase corrected using $\beta=0.076 \mathrm{mag} /{ }^{\circ}$ (left panel) and $\beta=$ $0.063 \mathrm{mag} /{ }^{\circ}$ (right panel) and arbitrarily located in the rotational phase.

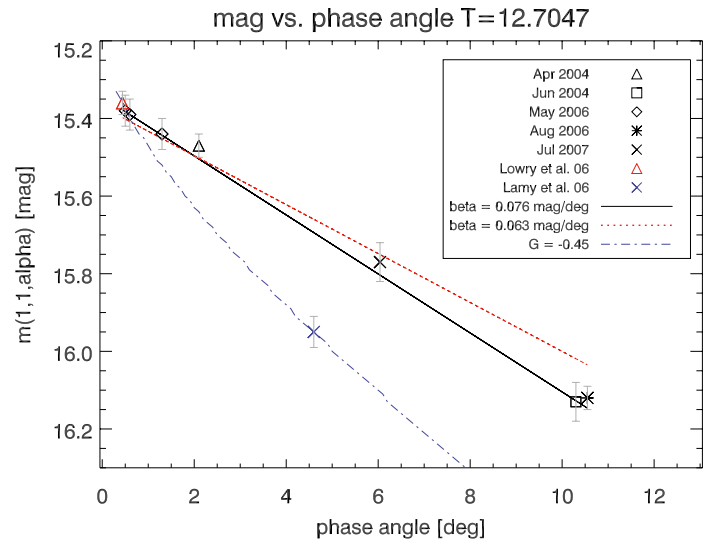

Fig. 6. Reduced magnitude vs. phase angle. The values correspond to the mid-point reduced magnitude in the $R$ filter. The different symbols correspond to the different datasets. In addition to our results the mid-point reduced magnitude from Lowry et al. (2006) and Lamy et al. (2006) are shown. The solid line indicates the slope of $\beta=0.076 \mathrm{mag} /{ }^{\circ}$, while the dotted one represents the slope of $\beta=0.063 \mathrm{mag} /{ }^{\circ}$. The blue dashed-dotted line represents the IAU-adopted phase law with $G=-0.45$ from Lamy et al. (2007).

solutions of group 1 , and $\beta=0.063 \mathrm{mag} /{ }^{\circ}$, representative, instead, of solutions belonging to group 2 , are overplotted to the data in Fig. 6. The steeper brightness dependence on phase angle $\left(\beta=0.076 \mathrm{mag} /{ }^{\circ}\right)$ seems to be agree better with the data.

The phase function determination requires observations at a different phase angle, but with constant aspect angle. This strict requirement makes its determination a difficult task, which is not really fulfilled by the available measurements, including ours. The nucleus phase function has been estimated for twelve JFCs, resulting in a mean value for the linear phase coefficient $\beta=$ $0.053 \pm 0.016 \mathrm{mag} /{ }^{\circ}$ (Snodgrass et al. 2011). The possible linear phase coefficient we obtain for $67 \mathrm{P} / \mathrm{C}-\mathrm{G}$ is slightly steeper than the one usually assumed for JFCs, closer to that of TNOs.

We cannot exclude that our linear phase coefficient of 67P/C$\mathrm{G}$ is affected by changes in the nucleus aspect angle, although the light curve profile of the comet did not change noticeably during the aphelion arc, when the phase function of the nucleus was sampled.

A previous determination of the phase function of $67 \mathrm{P} / \mathrm{C}-\mathrm{G}$ has been done by Lamy et al. (2007), who combined the HST (Lamy et al. 2006) and NTT (Lowry et al. 2006) observations. While at the time of the NTT observations $67 \mathrm{P} / \mathrm{C}-\mathrm{G}$ was at large heliocentric distance (at true anomaly between $151^{\circ}$ and $209^{\circ}$ ), Lamy et al. (2006) observed the comet in March 2003, a few months after its perihelion passage (at true anomaly of $\sim 106^{\circ}$ ). Thus, the orbital motion of the comet caused changes in the observing geometry. Assuming the IAU-adopted phase law as approximation of the phase function, Lamy et al. (2007) determined a slope parameter $G=-0.45$, which implies a very steep brightness dependence on phase angle, much steeper than the one we obtained, and a possible very strong opposition effect, never observed even for asteroids (Bowell et al. 1989; Tedesco 1989). Our observations of 67P/C-G do not confirm either of these results. We therefore believe that indeed aspect angle changes of the nucleus may be responsible for their deviating result.

In Fig. 6 we compare the mid-point reduced magnitude from our datasets with the one from Lowry et al. (2006) and Lamy et al. (2006). While the value from Lowry et al. (2006) agrees well with ours, the mid-point reduced magnitude from Lamy et al. (2006) is much lower. Most likely this discrepancy is caused by severe changes of the observing geometry.

\subsubsection{Surface colours and reflectance spectra of the nucleus}

The colour indices $V-R$ and $R-I$ were obtained from the April 2004 and July 2007 observations. In July 2007, thanks to observations also in the $B$ Bessell filter, we determined the $B-R$ colour index as well (see Table 3 ). The colour indices show that the nucleus of $67 \mathrm{P} / \mathrm{C}-\mathrm{G}$ is slightly redder than the Sun $\left((V-R)_{\odot}=0.36 \mathrm{mag},(R-I)_{\odot}=0.33 \mathrm{mag},(B-R)_{\odot}=1.03 \mathrm{mag}\right)$, as expected for cometary nuclei (Lamy et al. 2009; Snodgrass et al. 2008). $V, I$, and $B$ filter images of the comet were obtained at different rotational phases, thus the resulting colour indices sample different regions of the nucleus surface. Because the July 2007 dataset is phased differently depending on whether the rotational period used belongs to group 1 or to group 2 (Sect. 3.1.1), the rotational phase of the colour indices with regard to this dataset has the same dependence. Thus, we determined the rotational phase for both cases.

The values for the $V-R$ and $R-I$ indices are rather uniform (Fig. 7). For each couple of $V-R$ and $R-I$ colours in Table 3 we determined the mean spectral reddening (Delsanti et al. 2001) and found it to be constant within the error bars.

The average colour indices of the nucleus of $67 \mathrm{P} / \mathrm{C}-\mathrm{G}$ are summarised in Table 3 with the corresponding mean spectral reddening. Because observations in $B$ Bessell filter are also available, note that in July 2007 the mean spectral reddening was determined in the wavelength range 4360-7970 $\AA$ in addition to the one in the wavelength range $5450-7970 \AA$. The mean spectral reddening is the same for both wavelength ranges.

The nucleus spectrum of $67 \mathrm{P} / \mathrm{C}-\mathrm{G}$ was observed in July 2007; it complements the spectra obtained in May 2006, which are described in Tubiana et al. (2008). The spectrum is displayed in Fig. 8 (grey line) with the binned spectrum superimposed (binning to $70 \AA /$ pix). The $S / N$ ratios and the corresponding 


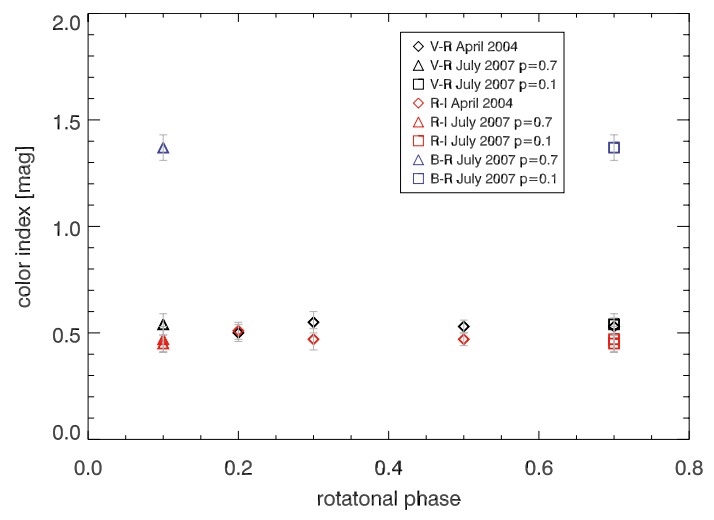

Fig. 7. Colour indices of $67 \mathrm{P} / \mathrm{C}-\mathrm{G}$ as determined from the photometric observations in April 2004 and July 2007 and summarised in Table 3.

relative errors in the binned spectrum at the different wavelengths are summarised in Fig. 8.

The spectrum does not show any absorption or emission feature and it displays reddening of rather constant slope of (12 \pm 1)\%/1000 $\AA$ in the wavelength range $4300-8500 \AA$. In the wavelength range $5000-8500 \AA$ the spectral reddening is marginally shallower of $(11 \pm 1) \% / 1000 \AA$.

Colour indices and the reflectance spectrum were obtained at different rotational phases, thus they sample different regions of the nuclear surface. They agree well (Fig. 8), which suggests that colour variations with the rotational phase are small, if present at all.

Lowry et al. (2006) determined a colour index $V-R=$ $(0.41 \pm 0.04) \mathrm{mag}$, slightly lower than those we obtained. It corresponds to a mean spectral reddening of $\sim 6 \% / 1000 \AA$, in the wavelength range 5500-6500 ̊. Lamy et al. (2006) claim a variation of the nucleus colour across the surface of the nucleus, a result which is not confirmed by our photometry and spectroscopy. Their averaged $V-R$ colour index $(0.52 \pm 0.05 \mathrm{mag})$ agrees with our results and corresponds to a photometric reflectivity gradient of $17 \% / 1000 \AA$ in the wavelength range 5500 $6500 \AA$, slightly higher than the reflectivity slope we obtained from our spectrum and photometry. Tubiana et al. (2008) determined colour indices $V-R=(0.38 \pm 0.04) \mathrm{mag}$ and $R-I=$ $(0.44 \pm 0.06) \mathrm{mag}$ in June 2004 and average colour indices $V-R=(0.54 \pm 0.03) \mathrm{mag}$ and $R-I=(0.47 \pm 0.03) \mathrm{mag}$ in May 2006, which correspond to a mean spectral reddening of $(5 \pm 3) \% / 1000 \AA$ and $(11 \pm 2) \% / 1000 \AA$, respectively, in the wavelength range 5450-7970 $\AA$. These results agree with our finding. Moreover, Tubiana et al. (2008) measured four visible spectra of the nucleus of $67 \mathrm{P} / \mathrm{C}-\mathrm{G}$ at different rotational phases, which do not show any absorption or emission feature and display a constant spectral reddening with rotational phase of $\sim(11 \pm 2) \% / 1000 \AA$, in the wavelength range $5450-7970 \AA$.

Because reflectance spectra provide a more detailed picture of the reddening behaviour, we favour our and Tubiana et al. (2008) conclusion of a uniform surface reflectance and colours of the nucleus of comet $67 \mathrm{P} / \mathrm{C}-\mathrm{G}$. The absence of gas emission features in the spectra is not surprising, because the comet is found without coma at these observing epochs. We cannot argue for a certain surface composition either, since no obvious absorption feature is found in our spectra.

\subsection{Tail-like structure}

Observations of $67 \mathrm{P} / \mathrm{C}-\mathrm{G}$ at various heliocentric distances (see Agarwal et al. 2007, 2010; Fulle et al. 2004, 2010; Ishiguro 2008; Tubiana et al. 2008) show a tail-like structure associated with the comet.

Owing to its low $S / N$ ratio, this structure usually cannot be detected in a single exposure. Thus, it is necessary to mediancombine images centred on the comet. This procedure not only increases the $S / N$ ratio of faint structures, but it also removes stars and other objects from the background, allowing an easier detection of low surface brightness features.

We detected the tail-like structure associated with comet 67P/C-G in April 2004 (Fig. 9).

Because of the insufficient number of images in $V$ and $I$ filter, we limited the search and characterisation of the tail-like structure to $R$ filter images only. We also determined the brightness profile as a function of the distance from the nucleus (Fig. 10). The peak located at $x=0^{\prime \prime}$ is the comet nucleus. On the righthand side of the nucleus, the brightness profile is systematically shifted with respect to the background level (dashed red line). This shift is caused by the presence of the tail-like structure.

In the July 2007 images we did not detect the tail-like structure. For the median combined image we estimated a $3 \sigma-$ detection limit of $27.5 \mathrm{mag} /{ }^{\prime \prime 2}$ for an extended object $25^{\prime \prime}$-wide, which can be used as an upper limit of the neck-line surface brightness in this observing epoch.

The tail-like structure associated with comet $67 \mathrm{P} / \mathrm{C}-\mathrm{G}$ was furthermore measured in the visible wavelength range in June 2004, and May 2006 images (Tubiana et al. 2008). At each observing epoch we determined its exact orientation and geometry, measuring its position angle (Table 4), its surface brightness, and its width.

We combined the April 2004, June 2004, May 2006, and July 2007 observations of the tail-like structure of $67 \mathrm{P} / \mathrm{C}-\mathrm{G}$ to investigate if it is better interpreted in terms of "old" dust trail or neckline. The "old" trail is composed of large dust particles emitted during the previous orbits of 67P/C-G, i.e. 1995/96 and earlier, while the neck-line is made of large dust particles emitted during the last orbit, i.e. 2002/03 apparition.

Geometric considerations: We compare the position angle of the tail-like structure as measured in the April 2004, June 2004, and May 2006 images $\left(\mathrm{PA}_{\text {measured }}\right)$ with:

1. The expected position angle of the "old" dust trail, as indicated by the direction of the heliocentric velocity vector projected in the sky $\left(\mathrm{PA}_{\mathrm{v}}\right)$.

2. The expected position angle of the neck-line, as predicted by dust tail simulations $\left(\mathrm{PA}_{\mathrm{NL}}\right)$ (Agarwal et al. 2010).

All aforementioned position angles are measured north over east and are summarised in Table 4.

The measured position angle of the tail-like structure agrees with those predicted by the dust tail simulations better than with the direction of heliocentric velocity vector projected in the sky, although deviations of the latter are less than one degree, but noticeable. Thus, based on geometrical considerations, we conclude that the tail-like structure is compatible with a neck-line, produced by the comet during the recent perihelion passage, and less likely an "old" dust trail.

Surface brightness versus heliocentric distance: We compare the measured surface brightness of the tail-like structure in April and June 2004, May 2006, and the upper limit in July 2007 with two models of the 67P/C-G dust production (Agarwal et al. 2010; Fulle et al. 2010). The model by Agarwal et al. (2010) 
Table 3. Colour indices of the 67P/C-G nucleus as determined from the photometric observations in April 2004 and July 2007.

\begin{tabular}{lccccc}
\hline \hline & $V-R[\mathrm{mag}]$ & $R-I[\mathrm{mag}]$ & $B-R[\mathrm{mag}]$ & $\phi$ & Reddening [\%/1000 $\AA]$ \\
\hline April 2004 & $0.50 \pm 0.04$ & $0.51 \pm 0.04$ & - & 0.2 & \\
& $0.55 \pm 0.05$ & $0.47 \pm 0.05$ & - & 0.3 & \\
& $0.53 \pm 0.03$ & $0.47 \pm 0.03$ & - & 0.5 & \\
& $0.53 \pm 0.04$ & - & - & 0.7 & $12 \pm 2$ \\
\hline average & $0.53 \pm 0.02$ & $0.49 \pm 0.02$ & - & & \\
\hline July 2007 & $0.54 \pm 0.05$ & $0.45 \pm 0.04$ & $1.37 \pm 0.06$ & $0.7^{\star}, 0.1^{\circ}$ & \\
\hline average & - & $0.47 \pm 0.06$ & - & $0.7^{\star}, 0.1^{\diamond}$ & \\
\hline
\end{tabular}

Notes. $\phi$ is the rotational phase at which the $V, I$, and $B$ filter images, which we used to determine the corresponding colour indices, were taken. July 2007 dataset: The rotational phase with regard to rotational periods belonging to group 1 is marked by *, while the one with regard to rotational periods belonging to group 2 is marked by ${ }^{\star}$. The average colour indices and the corresponding mean spectral reddening of $67 \mathrm{P} / \mathrm{C}-\mathrm{G}$ are shown. For April 2004 the mean spectral reddening was calculated in the wavelength range 5450-7970 A. In July 2007, in addition to the one relative to the wavelength range $5450-7970 \AA$ (marked by ${ }^{\bullet}$ ), thanks to observations also in $B$ filter we determined the mean spectral reddening also in the wavelength range 4360-7970 $\AA$ (marked by $\left.{ }^{\dagger}\right)$.

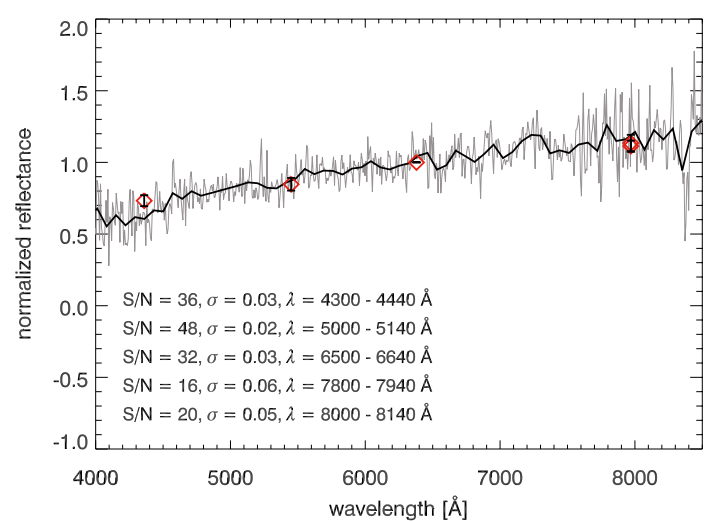

Fig. 8. Spectrum of $67 \mathrm{P} / \mathrm{C}-\mathrm{G}$ obtained in July 2007. The spectrum is normalised to unity at $6380 \AA$. In the original spectrum (grey line) the binned spectrum (black line) and the spectral reflectivities obtained from the photometric colour indices of the nucleus (diamonds) are superimposed. Signal-to-noise ratio $(S / N)$ and corresponding relative error $(\sigma)$ in the binned spectrum are reported with the wavelength range $(\lambda)$ used for their determination.

was fitted to three observations of the $67 \mathrm{P} / \mathrm{C}-\mathrm{G}$ trail and neckline at large heliocentric distances and therefore constrains only the properties of large particles (larger than $100 \mu \mathrm{m})$. Fulle et al. (2010) used observations distributed over a whole orbit of the comet, which allowed them to study the emission of smaller particles as well. The main difference between the two models is that Agarwal et al. (2010) assume that the dust production rates and size distribution are symmetric with respect to perihelion, with the dominant particle size in terms of light scattering crosssection being in the mm-range, while in the Fulle et al. (2010) model, the emission before perihelion is dominated by mm-sized particles, after perihelion by particles of the order of $10 \mu \mathrm{m}$. The dust ejection speeds in both models are similar at perihelion, but decrease more steeply in Agarwal et al. (2010), leading to lower predicted tail widths compared to Fulle et al. (2010).

According to both models, the dominant particles at the positions where our profiles were extracted had a radiation pressure parameter of $10^{-5}<\beta<3 \times 10^{-4}$. The parameter $\beta$ (Burns et al. 1979) denotes the ratio between solar gravity and radiation pressure force. This range corresponds to sizes of the order of millimetres to centimetres. For these particles, both models use a time-independent differential size distribution, described

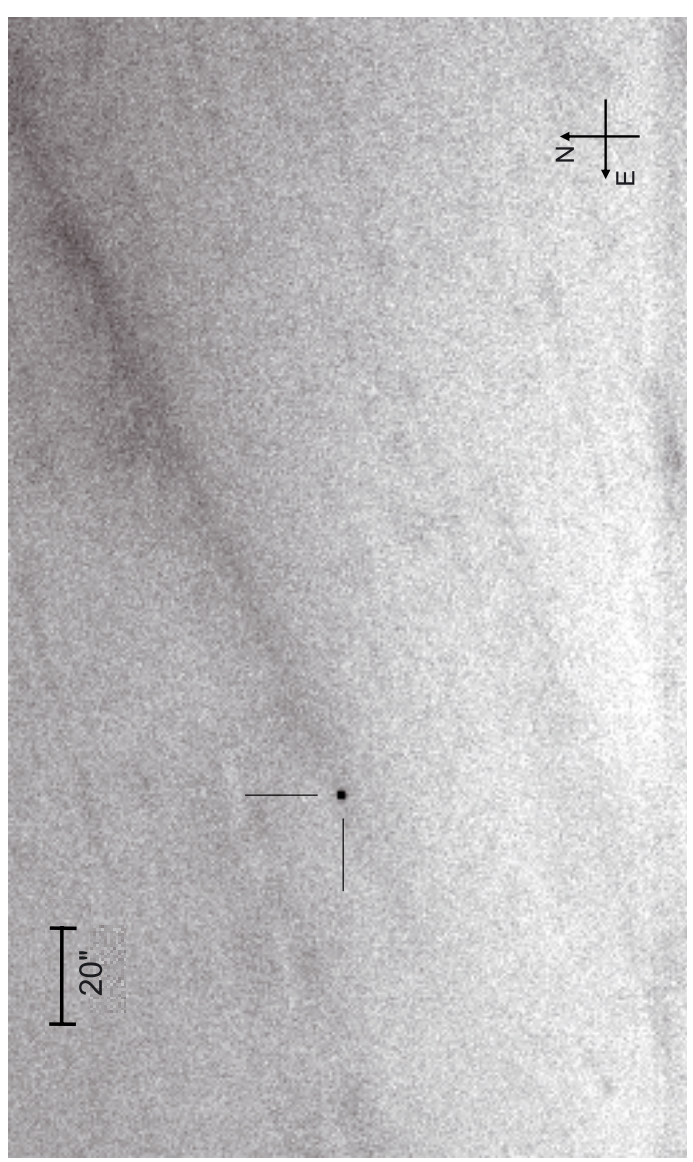

Fig. 9. Median-combined $R$ filter image of 67P/C-G taken in April 2004. The orientation of the image is shown and the position of the nucleus is marked. The tail-like structure at position angle $\mathrm{PA}=(295.7 \pm 0.2)^{\circ}$ has a projected extension of more than 143.7" and a width of about $25^{\prime \prime}$, corresponding to $\sim 3.9 \times 10^{5} \mathrm{~km}$ and $\sim 6.7 \times 10^{4} \mathrm{~km}$ at the comet distance, respectively.

by a power-law with the exponent of -4 . The production rates of these particles are roughly a factor of two higher in Agarwal et al. (2010).

We aimed to evaluate if the surface brightness variations are caused by dust dynamics or phase angle effects, because our observations were made at phase angles between $0.5^{\circ}$ and $10^{\circ}$ and it is likely that the light-scattering efficiency of the dust 
Table 4. Tail-like structure as measured in our images of 67P/C-G.

\begin{tabular}{lccccccc}
\hline \hline Date & $\mathrm{PA}_{\text {measured }}\left[{ }^{\circ}\right]$ & $\mathrm{SB}\left[\mathrm{mag} /{ }^{\prime \prime 2}\right]$ & $d\left[10^{5} \mathrm{~km}\right]$ & $l\left[10^{5} \mathrm{~km}\right]$ & $w\left[10^{4} \mathrm{~km}\right]$ & $\mathrm{PA}_{\mathrm{v}}\left[{ }^{\circ}\right]$ & $\mathrm{PA}_{N L}\left[^{\circ}\right]$ \\
\hline April 2004 & $295.7 \pm 0.2$ & $26.2 \pm 0.1$ & $\sim 1.3$ & $\sim 3.9$ & $\sim 6.7$ & 296.8 & $295.9 \pm 0.2$ \\
June 2004 & $298.1 \pm 0.2$ & $27.3 \pm 0.1$ & $\sim 1.5$ & $\sim 4.8$ & $\sim 2.5$ & 297.2 & $298.4 \pm 0.2$ \\
May 2006 & $285.6 \pm 0.2$ & $27.6 \pm 0.2$ & $\sim 3.8$ & $\sim 5.1$ & $\sim 2.5$ & 285.4 & $285.7 \pm 0.2$ \\
July 2007 & - & $>27.5$ & - & - & - & 279.0 & $279.8 \pm 0.3$ \\
\hline
\end{tabular}

Notes. $\mathrm{PA}_{\text {measured }}$ is its position angle measured north over east. The peak surface brightness (SB) of the tail-like structure is reported with the distance $d$ (in km at the projected distance of the comet) from the nucleus where the cross-cut to determine it was extracted, the length $l$ and the width $\mathrm{w}$ of the tail-like structure (in km projected distance at the comet). $\mathrm{PA}_{\mathrm{v}}$ is the direction of heliocentric velocity vector of the comet projected in the sky (from http://ssd.jpl.nasa.gov/horizons.cg) and $\mathrm{PA}_{\mathrm{NL}}$ is position angle of the neck-line as predicted by dust tail simulations.

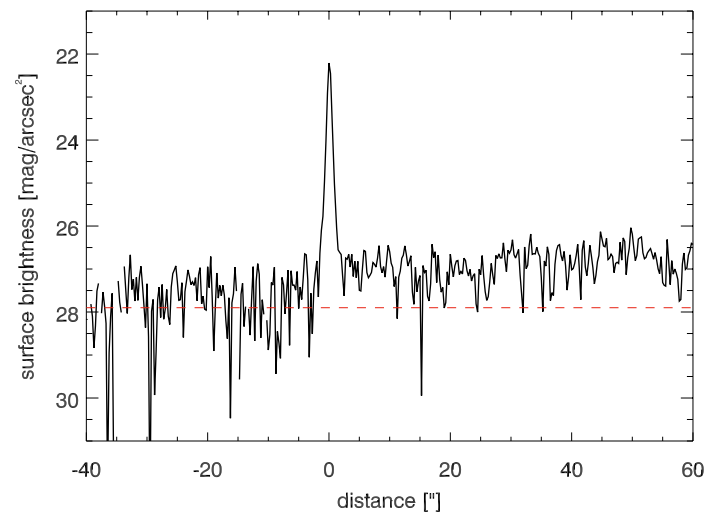

Fig. 10. Brightness profile of the tail-like structure of $67 \mathrm{P} / \mathrm{C}-\mathrm{G}$ as function of the distance from the nucleus. The peak located at $x=0^{\prime \prime}$ is the nucleus of the comet and the systematic shift in the profile for $x>0$ " is caused by the presence of the tail-like structure.

depends on the phase angle. To do this, we calculated the expected neck-line surface brightness without and with phase effect. In the first case we used a unit phase function $(\phi=1)$ and geometric albedo $p=0.04$. To our knowledge the phase function of mm-sized cometary grains has not been measured to date. Because these particles are much larger than the wavelength of the scattered sunlight, their phase function may be similar to that of the comet nucleus. Thus, to study the possible influence of the changing phase angle on our measured surface brightness, we used $\phi=\alpha \cdot \beta$, with $\beta=0.069 \mathrm{mag} /{ }^{\circ}$, the average phase coefficient derived in Sect. 3.1.1. In order to separate the impact of dust dynamics and phase function on the surface brightness of the tail-like structure, we display in Fig. 11 model calculations without phase effect (dashed lines) and with the nucleus phase function (solid lines).

The steep drop in surface brightness between April and June 2004 cannot be explained by particle dynamics alone. Both models with unit phase function predict a drop in surface brightness of $0.3 \mathrm{mag}$, caused by particles falling back behind the nucleus under the influence of radiation pressure and initial velocity. Smaller particles leave the field of view considered in the profile, while larger particles with a lower total cross-section replenish the images. The nucleus phase function predicts a brightness drop of 0.6 mag from April to June 2004. Both effects together reproduce the observations to a significant extent, but a difference of 0.3 mag remains unexplained. A possible interpretation is that the true dust phase function is even steeper than we assumed here, or that the models underestimate the fall-back speed of the dust. The April 2004 and May 2006 simulations are little affected by the uncertainty of the phase function, because of the low phase angles during these observations. The following

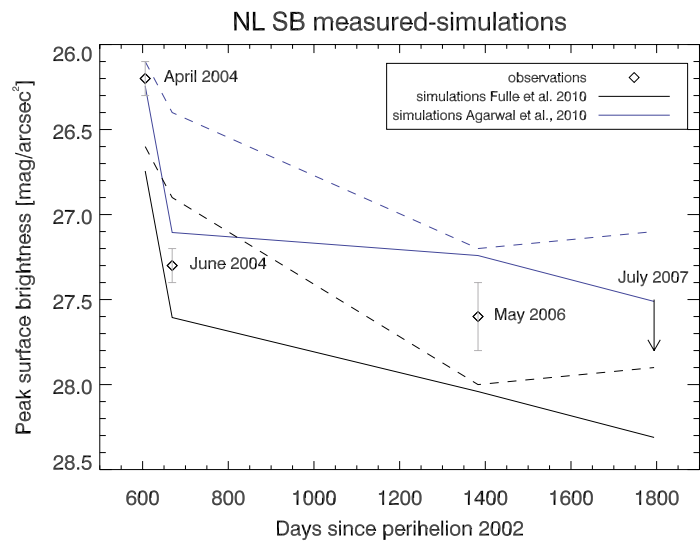

Fig. 11. Comparison between the surface brightness of 67P/C-G neckline, as measured in our images (diamonds) and that predicted by the dust models without phase effect (dashed lines) and with the nucleus phase function (solid lines).

discussion refers to the models in combination with the nucleus phase function. At all observing dates, the model of Fulle et al. (2010) tends to underestimate the observed dust light scattering cross-section, while the model of Agarwal et al. (2010) overestimates it. The general trend of decreasing surface brightness with time is supported by both models. Our non-detection of the taillike structure in July 2007 is consistent with the model-predicted flux, which is below the detection limit of the observation.

In both models, dust from the 2002/03 apparition contributes about $70 \%$ of the total cross-section in the tail-like structure in April 2004, decreasing to 30-40\% in July 2007. This is consistent with our analysis of the position angles that lead us to the conclusion that most of the dust we see in 2004-2006 was emitted during the 2002/03 apparition.

\section{Summary and conclusions}

We analysed broad-band images and low-resolution spectra obtained at the ESO VLT between April 2004 and July 2007, when $67 \mathrm{P} / \mathrm{C}-\mathrm{G}$ was at heliocentric distance of $r \geq 4.6 \mathrm{AU}$. The comet looks point-like, indicating that no coma was present around the nucleus. Because the comet does not show activity at large heliocentric distances, we conclude that $67 \mathrm{P} / \mathrm{C}-\mathrm{G}$ behaves as a fairly typical JFC. Moreover, we can infer that supervolatiles like $\mathrm{CO}$ do not play a role for distant activity. No observations of $67 \mathrm{P} / \mathrm{C}-\mathrm{G}$ from previous aphelion passages are available that can be compared with our results to evaluate whether changes in the comet's behaviour at large heliocentric distance took place during the last apparitions. Because the activity of $67 \mathrm{P} / \mathrm{C}-\mathrm{G}$ in the perihelion arc did not change significantly during the last orbital passages (Weiler et al. 2004; Schleicher 2006), this could 
suggest that also the comet's behaviour at large heliocentric distance does not change from one orbital revolution to the next. Thus, we could expect that during its approach to $67 \mathrm{P} / \mathrm{C}-\mathrm{G}$, ROSETTA will find the same conditions we detected during our observations.

The multiple solutions for the rotational period of $67 \mathrm{P} / \mathrm{C}-\mathrm{G}$, as determined from our observations, fall in the range $T_{\text {rot }}=$ $12.6801-12.8612 \mathrm{~h}$ and generally agree with those published by Lowry et al. (2006), who found $12.72 \pm 0.05 \mathrm{~h}$. Although a final conclusion on the accurate $T_{\text {rot }}$ cannot be drawn from our solutions, owing to insufficient sampling of the long time interval of the observation (1 year), our rotational period estimates have a much higher intrinsic precision. The rotational period $T_{\text {rot }}=12.41 \pm 0.41 \mathrm{~h}$, published by Lamy et al. (2006), is consistent with our results within its error, although by more than two orders of magnitude less accurate. By applying our procedures to the HST nucleus photometry of 67P/C-G (Lamy et al. 2006), we find plausible solutions for rotational periods of $6.1 \mathrm{~h}, 12.7$ $\mathrm{h}, 16.1 \mathrm{~h}$, and $18.4 \mathrm{~h}$. The solution $T_{\text {rot }}=12.7 \pm 0.2 \mathrm{~h}$, which corresponds to the one they published $\left(T_{\text {rot }}=12.41 \pm 0.41 \mathrm{~h}\right)$, agrees well with our results.

The bulk of the nuclei of ecliptic comets have rotational period between 5 and $18 \mathrm{~h}$ (Snodgrass et al. 2008), although the ensemble of JFCs measured may be biased towards short rotational periods. The possible rotational period we determined for the nucleus of $67 \mathrm{P} / \mathrm{C}-\mathrm{G}$ is in the aforementioned range. Thus, concerning the rotational period, $67 \mathrm{P} / \mathrm{C}-\mathrm{G}$ is a typical JFC.

With our observations we sampled the phase angle range between $0.5^{\circ}$ and $10^{\circ}$. We conclude that the linear phase function approximation is the one which better represents the measurements, compared to the IAU-adopted phase function. Indeed, the measurements obtained in April 2004 and July 2007, when $67 \mathrm{P} / \mathrm{C}-\mathrm{G}$ was at phase angle of about $\sim 2^{\circ}$ and $\sim 6^{\circ}$, respectively, show that the brightness dependence on phase angle is linear in the range between $0.5^{\circ}$ and $10^{\circ}$. This is an indication that $67 \mathrm{P} / \mathrm{C}-\mathrm{G}$ does not show a significant opposition effect. We determined that the magnitude dependence on the phase angle is very steep, with the linear phase coefficient in the range $\beta=0.061-0.076 \mathrm{mag} /{ }^{\circ}$. The determination of the phase function of $67 \mathrm{P} / \mathrm{C}-\mathrm{G}$ emphasises that the behaviour of some cometary nuclei at small phase angle might be different from that of asteroids, which display the opposition effect. This could indicate that comets display different light scattering properties compared to other small bodies in the solar system (i.e. asteroids).

The colour indices show that the nucleus of $67 \mathrm{P} / \mathrm{C}-\mathrm{G}$ is slightly redder than the Sun as expected for cometary nuclei (Lamy et al. 2009; Snodgrass et al. 2011). Moreover, the nuclear colours we determined are slightly redder than the mean colours determined for ecliptic comets: $\langle V-R\rangle=0.42 \mathrm{mag}$,
$<R-I>=0.38$ mag (Lamy et al. 2009). The absence of absorption or emission features in the spectra and a fairly constant spectral slope agrees with the results of Luu (1993), who obtained visible spectra of a small number of inactive comets. We iterate the conclusion that $67 \mathrm{P} / \mathrm{C}-\mathrm{G}$ appears to be a rather typical Jupiter family comet concerning its spectral behaviour in the visible wavelength range.

A tail-like structure was detected in April 2004, June 2004, and May 2006. Based on geometrical considerations and on surface brightness variations, the tail-like structure is better interpreted as a neck-line phenomenon instead of as an "old" dust trail.

Acknowledgements. We thank the astronomers and operation team of the VLT observatory for their skilled support during the visitor mode observations and for their careful work for our programmes in service mode. We also thank Rita Schulz (ESTEC/ESA), Gerhard Schwehm (ESTEC/ESA), and Jogy Stuewe (Leiden Observatory) for their support for the proposals. JLO acknowledges support from Spanish grants AYA2008-06202-C0301 and P07-FQM-02998.

\section{References}

Agarwal, J., Müller, M., \& Grün, E. 2007, Space Sci. Rev., 128, 79 Agarwal, J., Müller, M., Reach, W. T., et al. 2010, Icarus, 207, 992 Appenzeller, I., Fricke, K., Fürtig, W., et al. 1998, The Messenger, 94, 1 Bowell, E., Hapke, B., Domingue, D., et al. 1989, in Asteroids II, ed. R. P. Binzel, T. Gehrels, \& M. S. Matthews, 524-556

Burns, J. A., Lamy, P. L., \& Soter, S. 1979, Icarus, 40, 1

Delsanti, A. C., Böhnhardt, H., Barrera, L., et al. 2001, A\&A, 380, 347

Drahus, M., \& Waniak, W. 2006, Icarus, 185, 544

Fulle, M., Barbieri, C., Cremonese, G., et al. 2004, A\&A, 422, 357

Fulle, M., Colangeli, L., Agarwal, J., et al. 2010, A\&A, 522, A83

Hansen, K. C., Bagdonat, T., Motschmann, U., et al. 2007, Space Sci. Rev., 128, 133

Ishiguro, M. 2008, Icarus, 193, 96

Kelley, M. S., Wooden, D. H., Tubiana, C., et al. 2009, AJ, 137, 4633

Kidger, M. R. 2004, A\&A, 420, 389

Lamy, P. L., Toth, I., Weaver, H. A., et al. 2006, A\&A, 458, 669

Lamy, P. L., Toth, I., Davidsson, B. J. R., et al. 2007, Space Sci. Rev., 128, 23

Lamy, P. L., Toth, I., Weaver, H. A., A'Hearn, M. F., \& Jorda, L. 2009, A\&A, 508, 1045

Lowry, S. C., Fitzsimmons, A., Jorda, L., et al. 2006, in BAAS, 38, 492

Luu, J. X. 1993, Icarus, 104, 138

Michalowski, T. 1988, Acta Astron., 38, 455

Schleicher, D. G. 2006, Icarus, 181, 442

Schwehm, G., \& Schulz, R. 1998, in Laboratory astrophysics and space research, ed. P. Ehrenfreund, C. Krafft, H. Kochan, \& V. Pirronello, Astrophys. Space Sci. Libr., 236, 537

Snodgrass, C., Lowry, S. C., \& Fitzsimmons, A. 2008, MNRAS, 385, 737

Snodgrass, C., Fitzsimmons, A., Lowry, S. C., \& Weissman, P. 2011, MNRAS [arXiv: 1101.4228]

Tedesco, E. F. 1989, in Asteroids II, ed. R. P. Binzel, T. Gehrels, \& M. S. Matthews, 1090

Tubiana, C., Barrera, L., Drahus, M., \& Böhnhardt, H. 2008, A\&A, 490, 377

Weiler, M., Rauer, H., \& Helbert, J. 2004, A\&A, 414, 749 